\title{
Role of obstetric colour doppler studies in predicting perinatal outcome in pregnancy induced hypertension
}

\author{
Ritu Mishra ${ }^{1}$, Aditya P. Misra ${ }^{2 *}$
}

\begin{abstract}
${ }^{1}$ Department of Obstetrics and Gynecology, Rama Medical College Hospital and Research Centre, Kanpur, Uttar Pradesh, India

${ }^{2}$ Department of Radiodiagnosis, Rama Medical College Hospital and Research Centre, Kanpur, Uttar Pradesh, India
\end{abstract}

Received: 16 December 2019

Revised: 16 January 2020

Accepted: 24 January 2020

\section{*Correspondence:}

Dr. Aditya P. Misra,

E-mail: saggimishra@hotmail.com

Copyright: ( ) the author(s), publisher and licensee Medip Academy. This is an open-access article distributed under the terms of the Creative Commons Attribution Non-Commercial License, which permits unrestricted non-commercial use, distribution, and reproduction in any medium, provided the original work is properly cited.

\section{ABSTRACT}

Background: Pregnancy induced hypertension (PIH) is associated with adverse perinatal outcome. Multi vessel colour Doppler studies are useful in these cases for timely intervention. The aim of present study was to know the significance of umbilical, middle cerebral and uterine artery Doppler in PIH and to analyse their role in predicting perinatal outcome.

Methods: This is a study of 150 pregnant women with pregnancy induced hypertension (PIH). These patients were evaluated with colour Doppler and were followed subsequently for any adverse perinatal outcome.

Results: Out of 150 cases $70 \%$ of cases were found in 20-30 years age group. 98 cases had abnormal uterine artery Doppler indices accounting for $65.3 \%$, while 52 cases had a normal Doppler index accounting for $34.3 \%$. Out of 150 cases, 94 (64.6\%) cases had abnormal middle cerebral artery. In our study 53 cases had abnormal umbilical artery Doppler indices accounting for 35.4\%, while 97 cases had a normal Doppler index accounting for 64.6\%. Out of 150 cases in our study 27 cases had Reversal of a wave in ductus venosus waveform that is in $18 \%$. In this study total 5 cases $(35.7 \%)$ of perinatal mortality were seen.

Conclusions: The knowledge of various doppler parameters may help to improve pregnancy outcome and identification of PIH at earliest gestation age as compared to other antepartum test modalities.

Keywords: Colour doppler, Fetoplacental, Perinatal mortality, Pre-eclampsia, Pregnancy induced hypertension, Uteroplacental flow

\section{INTRODUCTION}

Hypertensive disorders during pregnancy remain the most common medical complications, leading to a majority of adverse perinatal and maternal outcome, despite numerous efforts that are made at early diagnosis, prevention and treatment of PIH. The incidence of various hypertensive disorders of pregnancy varies widely from 5 to $15 \%$ with perinatal mortality of 15 to $20 \%$ in the developing countries. ${ }^{1,2}$ Pregnancy induced hypertension includes gestational hypertension, pre- eclampsia and eclampsia. It has many complications, most common are Intra uterine growth retardation, fetal distress, Apgar score below 7 at 5 minutes, admission to neonatal intensive care unit and perinatal deaths including intrauterine and early neonatal deaths. Other complications include hypoxic ischemic encephalopathy, intraventricular haemorrhage, pulmonary haemorrhage and necrotizing enterocolitis. Maternal complication included caesarean delivery due to fetal distress, convulsions, pulmonary oedema, HELLP syndrome, rarely maternal mortality. The main goals of prenatal 
testing are to identify fetus at increased risk for perinatal morbidity and mortality. Early detection of disease would lead to an improved outcome, through increased surveillance and use of prophylactic therapies such as low dose aspirin. ${ }^{3}$ The placenta through implantation and development modifies the uterine circulation from one of low flow and high resistance to one of high flow and low resistance. The primary defect that predisposes pregnancy to uteroplacental complication appear to be partial/complete failure of trophoblastic invasion. ${ }^{4}$ It is therefore desirable to know the accurate changes in uteroplacental and fetal circulation to predict perinatal outcome hence to plan appropriate intervention. The abnormal vascular resistance to blood flow in the uteroplacental and fetoplacental circulation can be well studied by Doppler ultrasound. Colour Doppler studies provides a repetitive, non-invasive tool for hemodynamic monitoring of fetal well-being. ${ }^{5}$ These vascular Doppler analyses predominantly plays role in decision making process in high risk obstetric cases. ${ }^{6,7}$ Several studies suggested a significant decrease in neonatal morbidity and mortality when the rates of preterm birth, growth retarded foetuses and perinatal death, are significantly increased in pregnancies complicated by severe preeclampsia. ${ }^{8,9}$ Elective preterm delivery is frequently advised for hypertensive women in an attempt to improve neonatal outcome, although this may result in higher fetal mortality and morbidity because of prematurity.

\section{METHODS}

\section{Patient selection and clinical presentation}

This is a prospective study of 150 pregnant women with pregnancy induced hypertension (PIH) evaluated with colour Doppler and followed subsequently for any adverse perinatal outcome. The study was conducted in department of radiodiagnosis, Rama Medical College Hospital and Research Centre, Kanpur over a period of July 2018 to august 2019.

\section{Inclusion criteria}

Women with pregnancy induced hypertension with more than 24 weeks of pregnancy were included in the study. Acceptable reference standards for pregnancy induced hypertension were persistently high systolic $(\geq 140$ $\mathrm{mmHg}$ ) or diastolic ( $\geq 90 \mathrm{mmHg}$ ) blood pressure with or without proteinuria $[\geq 0.3 \mathrm{~g}$ of protein in 24 hour urine collection, or dipstick test result of $\geq 1+$ (equivalent to $30 \mathrm{mg} / \mathrm{dL}$ in single urine sample)] of new onset after 20 weeks of gestation.

\section{Exclusion criteria}

Pregnancy with severe congenital anomaly was excluded from study. In the PIH group the age of subjects ranged from 19-33 years with their gestational age at the time of examination varying from 25 weeks to 39 weeks.
All the subjects had their gestational age confirmed by 2$\mathrm{D}, \mathrm{B}$ mode ultrasonography by estimation of BPD (Biparietal diameter), FL (femur length) and abdominal circumference (AC) of the fetus, and then they were studied by colour Doppler. The findings at the time of first examination were taken into consideration. Repetitive Doppler studies were performed. The subjects were followed till delivery and data at the time of delivery and final perinatal outcome was collected.

\section{Technical consideration}

1. Color Doppler ultrasound unit - A Siemens Sonoline G - 50

2. Insonation angle was taken less than $60 \mathrm{deg}$. And pre-systolic and end-diastolic velocities were defined manually after observing at least three consecutive waves.

\section{Technique of scanning}

Doppler measurements were taken with the mother in recumbent position during fetal inactivity and apnoea. Gestational age of the patient was obtained using B mode 2-D 3.5-5 MHz convex probe and Doppler examination was done by pulsed Doppler method. The BPD (bi parietal diameter), FL (femur length) AC (abdominal circumference) and MGA (mean gestational age) and other relevant data were obtained initially. Then colour Doppler was done.

\section{Uterine artery}

The uterine artery was studied by first identifying the placental site. If the placenta was unilateral, uterine artery of that side was studied. In case of central placenta, both uterine arteries were evaluated. The main branch of uterine artery is located at cervicocorporal junction. Uterine artery was examined at the level of internal os with full bladder seen crossing perpendicular to external iliac artery. Doppler velocimetry measurement performed near this location. The probe was positioned to obtain the best quality signals from uterine artery recognized by its characteristic sound and flow pattern. When the screen showed several waveforms of similar height and pattern, one image was frozen and peak systolic and end diastolic velocities were marked with cursors. The resistive index, pulsatility index and S/D ratio were obtained and the value was noted down. Uterine artery flow waveforms were considered abnormal with the presence of early diastolic notch and S/D ratio of more than 2.6 in any one either right or left uterine artery.

\section{Umbilical artery}

Free - floating loop of umbilical cord was examined to evaluate Umbilical artery. Values at mid cord or placental insertion were taken as they were clinically reliable. Umbilical artery S/D ratio was considered abnormal when it was more than 3 after 30 weeks' gestation. 
Umbilical artery RI and PI were considered abnormal when it was more than the 95th percentile of the range of reference. Absent and reverse end diastolic flow of umbilical artery Doppler were considered abnormal

\section{Middle cerebral artery}

Middle cerebral artery was located in a transverse section of fetal skull at the level of thalami and cavum septum pellucidum. A transverse view of the fetal brain was obtained at the level of the biparietal diameter. The transducer is then moved wing of the sphenoid bone. Using color flow imaging, the middle cerebral artery can be seen as a major lateral branch of the circle of Willis, running anterolateral at the borderline between the anterior and the middle cerebral fossae. The pulsed Doppler sample gate is then placed on the middle portion of this vessel to obtain flow velocity waveforms. During the study care was taken not to take readings during fetal breathing for gross body movements and to apply minimal pressure to the maternal abdomen with the transducer, as fetal head compression is associated with alterations of intracranial arterial waveform. When the screen shows several waveforms of similar height and pattern, one image was frozen and peak systolic and end diastolic velocities were marked with cursors. Middle cerebral artery RI and PI were considered abnormal when it was more than the 95th percentile of the range of reference.

\section{Cerebroplacental ratio or MCA / UA PI ratio}

This Doppler index is useful in identifying fetuses with increased placental and/ or decreased cerebral resistance.

\section{Ductus venosus}

The ductus venosus could be visualized either in a midsagittal longitudinal plane of the fetal trunk or in an oblique transverse plane through the upper abdomen. The sample volume was positioned at its origin from the umbilical vein, where colour Doppler indicated the highest velocities. On gray scale DV is identified as a small vessel connecting $\mathrm{UV}$ and IVC. On $\mathrm{C} / \mathrm{F}$ it is the brightest vessel showing aliasing (d/t turbulent flow) situated anterior to fetal aorta. Follow up Doppler studies were performed if indicated to determine and monitor a favorable or worsening fetal wellbeing status. However only the results of last Doppler ultrasound within one week of delivery were used for analysis. Outcome of pregnancy was studied in terms of mode of delivery, preterm or term delivery, Intra uterine growth retardation, birth weight, Apgar score, Intra uterine fetal death, and perinatal mortality.

\section{RESULTS}

PIH cases were distributed under the age group of $<20$, $20-25,25-30,30-35$ years. majority of the cases came under the age group of 20-25 accounting for about $36.7 \%$
Mean age of subjects being 25.7 years. $33.3 \%$ cases were found in 25-30 age group. Thus 70\% of cases were found in patients of 20-30 years of age (Table 1).

Table 1: Distribution of PIH cases according to age.

\begin{tabular}{|lll|}
\hline Age group & Total cases & $\%$ \\
\hline$<20$ & 7 & $4.67 \%$ \\
\hline $20-25$ & 55 & $36.7 \%$ \\
\hline $25-30$ & 50 & $33.3 \%$ \\
\hline $30-35$ & 38 & $25.3 \%$ \\
\hline Total & 150 & $100 \%$ \\
\hline
\end{tabular}

Table 2: Distribution of PIH cases according to parity.

\begin{tabular}{|lll|}
\hline Parity & No. of cases & $\%$ \\
\hline 0 & 62 & $41.4 \%$ \\
\hline 1 & 66 & $43.9 \%$ \\
\hline 2 & 18 & $12.1 \%$ \\
\hline 3 & 4 & $2.43 \%$ \\
\hline Total & 150 & $100 \%$ \\
\hline
\end{tabular}

This (Table 2) shows distribution of PIH cases according to parity, $62(41.4 \%)$ patients were nullipara, $66(43.9 \%)$ were primipara. Rest of $14.53 \%$ cases were multipara.

Table 3: Distribution of cases under normal and abnormal uterine artery indices.

\begin{tabular}{|lll|}
\hline Uterine artery indices & Total cases & $\%$ \\
\hline Bilateral abnormal & 56 & $37.3 \%$ \\
\hline Unilateral abnormal & 42 & $28 \%$ \\
\hline Normal & 52 & $34.7 \%$ \\
\hline Total & 150 & $100 \%$ \\
\hline
\end{tabular}

Among total PIH cases, 98 cases had abnormal uterine artery Doppler indices accounting for $65.3 \%$, while 52 cases i.e., $34.5 \%$ had a normal Doppler indices. Out of 98 cases of PIH showing raised Doppler indices and diastolic notch, 56 cases showed bilateral abnormality (B), either raised indices, diastolic notch or both leading to $37.3 \%$ cases of total. Whereas, 42 cases that is $28 \%$ cases showed unilateral Doppler abnormality (Table 3 ).

Table 4: Distribution of cases under normal and abnormal middle cerebral artery indices.

\begin{tabular}{|lll|}
\hline Waveform-MCA & Total cases & $\%$ \\
\hline I-increase diastolic flow & 95 & $64.6 \%$ \\
\hline N-normal & 55 & $36.7 \%$ \\
\hline Total & 150 & $100 \%$ \\
\hline
\end{tabular}

In our study, out of 150 cases, 95 cases (64.6\%) had abnormal middle cerebral artery Doppler indices showing increased diastolic flow with brain sparing effect while 55 cases had normal Doppler indices accounting for $36.7 \%$ (Table 4). 
Table 5: Distribution of cases under normal and abnormal umbilical artery indices.

\begin{tabular}{|lll|}
\hline Waveform - UA & Total cases & $\%$ \\
\hline Normal & 97 & $64.6 \%$ \\
\hline Decreased diastolic flow (D) & 42 & $28 \%$ \\
\hline Absent diastolic flow (A) & 4 & $2.7 \%$ \\
\hline Reversal of diastolic flow (R) & 7 & $4.7 \%$ \\
\hline Total & 150 & $100 \%$ \\
\hline
\end{tabular}

According to this Table 5 out of 150 PIH cases, 53 cases had abnormal umbilical artery Doppler indices i.e., $35.4 \%$, while $97(64.6 \%$.) cases had normal Doppler indices. Out of 53 cases of PIH showing raised Doppler indices, 42 cases showed just decreased diastolic flow. While four cases showed absent diastolic flow (A) making it $2.7 \%$ of cases. 7 cases showed Reversal of diastolic flow (R) that is $4.7 \%$ of total cases.
Table 6: Distribution of cases with reversal of a wave in ductus venusus.

\begin{tabular}{|lll|}
\hline Ductus venuses wave & Total cases & $\%$ \\
\hline A - reversal of a wave & 27 & $18 \%$ \\
\hline N - normal & 123 & $82 \%$ \\
\hline Total & 150 & $100 \%$ \\
\hline
\end{tabular}

The Table 6 revealed that out of 150 cases in our study 27 $(18 \%)$ cases had reversal of a wave in ductus venusus waveform, while $123(82 \%)$ cases had a normal ductus venusus Doppler waveform.

In our study, out of 51 cases with bilateral uterine artery abnormality (B) maximum cases i.e. 44 cases $(86 \%)$ were low birth weight, with $15(29.4 \%)$ cases with birth weight $<1000 \mathrm{gm}$. As compared to this only 7 cases $(13.72 \%)$ of birth weight $>2500 \mathrm{gm}$ (Table 7).

Table 7: Correlation of birth weight with abnormal and normal uterine artery indices.

\begin{tabular}{|llllll|}
\hline Birth weight (In gms) & $<\mathbf{1 0 0 0}$ & $\mathbf{1 0 0 0 - 1 5 0 0}$ & $\mathbf{1 5 0 0 - 2 5 0 0}$ & $>\mathbf{2 5 0 0}$ & Total \\
\hline B - bilateral abnormal & $15(29.4 \%)$ & $7(13.72 \%)$ & $22(43.13 \%)$ & $7(13.72 \%)$ & 51 \\
\hline N - normal & $0(0 \%)$ & $7(12.72 \%)$ & $15(27.27 \%)$ & $33(60 \%)$ & 55 \\
\hline U - unilateral abnormal & $7(15.90 \%)$ & $0(0 \%)$ & $22(50 \%)$ & $15(34.09 \%)$ & 44 \\
\hline Total & 22 & 14 & 59 & 55 & 150 \\
\hline
\end{tabular}

Table 8: Perinatal mortality in relation to abnormal uterine artery indices.

\begin{tabular}{|c|c|c|c|}
\hline \multirow{2}{*}{ Uterine artery } & \multicolumn{2}{|l|}{ Outcome } & \multirow{2}{*}{ Total } \\
\hline & LB & IUD & \\
\hline B - bilateral abnormal & $33(65 \%)$ & $18(35 \%)$ & $51(100 \%)$ \\
\hline $\mathrm{N}$ - normal & $55(100 \%)$ & $0(0 \%)$ & $55(100 \%)$ \\
\hline U - unilateral abnormal & $37(84 \%)$ & $7(16 \%)$ & $44(100 \%)$ \\
\hline Total & $125(83 \%)$ & $25(17 \%)$ & $150(100 \%)$ \\
\hline
\end{tabular}

$\chi^{2}$ test was applied and association between $\mathrm{B} / \mathrm{L}$ abnormal uterine artery and poor perinatal outcome in form of extremely low birth wt. was found to be statistically significant, $\chi^{2}=4.97$ ( $\mathrm{p}$ value $<0.05$ ). While in normal uterine artery $(\mathrm{N})$, out of 55 cases maximum cases i.e., 33 $(60 \%)$ were of birth weight $>2500 \mathrm{gm}$ (normal) with none of the cases with birth weight $<1000$ gm. 6 cases (40\%) had weight between 1000-2500 gm. Out of 44 cases with unilateral uterine artery abnormality (U) 7 $(15.9 \%)$ cases had birth weight $<1000$ gm. 22 cases (50\%) were low birth weight, between 1500-2500 gm. And 15 cases (34.09\%) were of normal weight (Table 8)

In our study, out of 51 cases with bilateral uterine artery abnormality (B) there were 10 intrauterine deaths, 5 cases had birth asphyxia and were admitted to NICU but died on third postnatal day. Another 3 cases developed severe neonatal jaundice and died on tenth postnatal day leading to total 18 cases $(35 \%)$ of perinatal mortality.
In this study $\chi^{2}$ test was applied and association between $\mathrm{B} / \mathrm{L}$ abnormal uterine artery and perinatal mortality was found to be statistically significant, $\chi^{2}=6.47$ ( $\mathrm{p}$ value < $0.05)$. Out of 44 cases with unilateral uterine artery abnormality (U) there were 7 IUD (16\% of cases) and 37 live births (84\%) (Table 9).

\section{DISCUSSION}

The role of Doppler ultrasound to understand the uteroplacental and fetoplacental circulation in pregnancy induced hypertension is well studied by various researchers. In a normal pregnancy, there is a low resistance in uteroplacental and fetoplacental circulation. Pregnancy induced hypertension is associated with defective trophoblastic invasion which further leads to abnormal placental vascular flow. Doppler studies help in detecting the abnormal vascular resistance patterns in compromised fetuses so that timely intervention can be done. The mean age of the mother in present study is 25.7 
years. In similar studies by Smitha et al and Ozeren et al, they reported mean age as 23.4 and 27.6 years respectively. ${ }^{10,11}$ In the present study $43.5 \%$ of the patients were primigravidas where as in the studies by Khalid $\mathrm{M}$ et al and Lakhkar BN $77.7 \%$ and $60.3 \%$ patients were primigravida respectively. ${ }^{12,13}$ In our study uterine artery, umbilical artery, middle cerebral artery and ductus venosus are studied to improve perinatal outcome. In pregnancy induced hypertension there is inadequate invasion of spiral arteries leading to increased resistances in spiral arteries. This leads to increased impedance of blood flow in uterine arteries by Fleischer A, Schulman $\mathrm{H}$, Farmakides $\mathrm{G}$ et al. ${ }^{14}$ The findings in our study are consistent with above. In our study of uterine artery Doppler velocimetry, among 150 cases, 52 (37.3\%) subjects had normal flow pattern in uterine artery and 98 $(65.3 \%)$ had abnormal flow pattern with raised indices and diastolic notches. Out of 98 cases of PIH showing raised Doppler indices and diastolic notch, 56 cases showed bilateral abnormality (B), either raised indices, diastolic notch or both leading to $(37.3 \%)$ cases. Our findings were consistent with Khalid $\mathrm{M}$ et al, to determine the role of color Doppler sonography in evaluation of fetal outcome in 58 antenatal females (22 normotensives, 36 hypertensive) in their third trimester of pregnancy. ${ }^{12}$ Arteries evaluated included - bilateral uterine arteries, umbilical artery, fetal middle cerebral and fetal aorta. In his study, $34(94.44 \%)$ out of 36 hypertensive patients showed abnormal uterine artery flow. U/L Uterine artery involved in 12 cases $(33.3 \%)$. $\mathrm{B} / \mathrm{L}$ uterine artery was involved in $22(61.11 \%)$ cases. According Jackson MR et al, patients with uterine artery notches and high resistance flow had significantly higher rates of fetal growth retardation and caesarean delivery because of fetal distress and had significantly bad pregnancy outcome. ${ }^{15}$ In our study of umbilical artery among 150 patients, $53(35.4 \%)$, cases had abnormal umbilical artery Doppler indices while 97 (64.6\%) cases had a normal Doppler indices. In our study of middle cerebral artery Doppler velocimetry among 150 case group, 95 (64.6\%) had abnormal Middle cerebral artery. Doppler showing increased diastolic flow with brain sparing effect (I) while $55(36.7 \%)$ cases had normal middle cerebral artery Doppler indices. Similar findings were seen in study by Lakhkar BN et al prospectively examined 58 singleton pregnancies beyond 30 weeks of gestation complicated by intrauterine growth restriction and severe pre-eclampsia. ${ }^{13}$ Thirty-six patients of the 58 included in the study population had at least one major or minor adverse outcome. Chan et al, studied 71 high risk fetuses with weekly UA and MCA Doppler US examinations until delivery. ${ }^{16}$ In $15.5 \%$ (11 of 71) of fetuses, there was perinatal mortality or major morbidity, including major intracranial haemorrhage, periventricular leukomalacia, necrotizing enterocolitis, and major neurologic handicap (follow-up data in 24 cases and up to only 2 years of age). By using the last Doppler USG result for analysis, the UA/MCA resistance index ratio, compared with the UA systolic-to-diastolic ratio, was more sensitive $(75 \%$ versus $64 \%)$ but less specific $(60 \%$ versus 74\%). UA Doppler USG was a better predictor for each of the individual adverse outcomes when separate analyses were performed. Bhatt CJ, Arora J studied total of 100 cases of PIH between 28 -36 weeks of gestation over a period of 2 years. ${ }^{17}$ For studying uterine, umbilical and fetal middle cerebral arteries, S/D ratio of greater than 3 and 2.6 in umbilical and uterine arteries respectively were considered abnormal Out of $100,11 \%$ cases out of these have both uterine and umbilical arteries as abnormal, $56 \%$ had abnormal S/D ratio in umbilical artery and/or uterine artery $60 \%$ of these patients delivered IUGR babies. In patients with absent end diastolic velocity (AEDV) and reversed end diastolic velocity (REDV) perinatal mortality was 50\% and $50 \%$ had IUGR babies. The fetuses with compromised circulation showed increased diastolic flow in fetal MCA suggestive of brain sparing effect. In our study of Ductus venous velocimetry of 150 case group, 123 (82\%) have normal flow whereas $27(18 \%)$ had abnormal flow with Reversal of ' $a$ ' wave in Ductus Venosus Waveform (A) Turan OM et al, in 177 study participants underwent a total of 1069 examinations. ${ }^{18}$ Days of duration of absent/reversed UA end-diastolic velocity (UA-AREDV), low middle cerebral artery PI (brain sparing), ductus venosus (DV) and umbilical vein Doppler abnormalities were related to stillbirth, major neonatal morbidity and intact survival. The duration of an absent/reversed a-wave in the DV (DV-RAV) was significantly higher in stillbirths (median, 6 days) compared with intact survivors and those with major morbidity (median, 0 days for both; $p=0.006$ and $p=0.001$, respectively). Baschat AA et al, patients with suspected intrauterine growth restriction (IUGR) underwent uniform fetal assessment including umbilical artery (UA), ductus venosus (DV) and umbilical vein (UV) Doppler. ${ }^{16}$ Absent or reversed UA end-diastolic velocity (UA-AREDV), absence or reversal of atrial systolic blood flow velocity in the DV (DV-RAV) and pulsatile flow in the umbilical vein (PUV) were examined for their efficacy to predict critical outcomes (stillbirth, neonatal death, perinatal death, acidemia and birth asphyxia) before 37 weeks' gestation Overall, DVRAV or P-UV offered the best prediction of academia and neonatal and perinatal death irrespective of the UA waveform. In fetuses with UA-AREDV, prediction of asphyxia and stillbirth was significantly enhanced by venous Doppler. Prediction of critical perinatal outcomes is improved when venous and umbilical artery qualitative waveform analysis is combined. The incorporation of venous Doppler into fetal surveillance is therefore strongly suggested for all preterm IUGR fetuses.

\section{CONCLUSION}

Doppler ultrasound offers the ability to screen cases of pregnancy induce hypertension early in gestation with a non-invasive haemodynamic testing mechanism. Doppler indices from the fetal circulation can reliably predict adverse perinatal outcome in an obstetric patient in high risk population. The knowledge of uterine and umbilical 
artery waveform may help to improve pregnancy management and any permit identification and assessment of pregnancy induced hypertension at earliest gestation age as compared to other antepartum test modalities. Early identification creates possibility of early intervention and therapy. Thus, multi vessel color Doppler ultrasound studies are important monitoring tools in patients with PIH to identify compromised fetuses in utero and to take timely appropriate action.

\section{Funding: No funding sources}

Conflict of interest: None declared

Ethical approval: The study was approved by the Institutional Ethics Committee

\section{REFERENCES}

1. Cunnigham FG, Leveno KJ, Bloom SL, Hauth JC, Rouse DJ, Spong CY; Pregnancy Hypertension, Willams Obstetrics. $23^{\text {rd }}$ Edition, McGraw-Hill, New York, 2010;706-756.

2. National high blood pressure education program working group. Report of the National High Blood Pressure Education Program working group on High Blood Pressure in pregnancy. Am J Obstet Gynecol. 2000;183:1-22.

3. Mc Parland, Peare P, Chamberlain JM. Doppler sonography and aspirin in prevention of pregnancy induced hypertension. Lancet. 1990;335(8705):15525.

4. DeWolf KT, Robertson F. Inadequate maternal vascular response to placentation in pregnancies complicated by pre-eclampsia. Obstet Gynaecol. 1986;93(10):1049-59.

5. Carroll BA. Duplex Doppler systems in obstetric ultrasound. Radiol Clin N Am. 1990;25(1):189-202.

6. Hoffman C, Galan HL. Assessing the 'at-risk' fetus: Doppler ultrasound. Curr Opin Obstet Gynaecol. 2009;21:161-6.

7. Fleischer A, Schulman H, Farmakides G, Bracero L, Grunfeld L, Rochelson B, et al. Uterine artery Doppler velocimetry in pregnant women with hypertension. Am J Obstet Gynecol. 1986;154(4):806-13.

8. Lin CC, Lindheimer MD, River P. Fetal outcome in hypertensive disorders of pregnancy. Am J Obstet Gynecol. 1982;142:255.

9. Sibai B, Spinnato JA, Watson DL. Pregnancy outcome in 303 cases with severe preeclampsia. Obstet Gynecol. 1984;64:319-21.
10. Smitha K, Sowmya K, Malathi T. Study of Doppler waveforms in pregnancy induced hypertension and its correlation with perinatal outcome. Int $\mathbf{J}$ Reprod Contracept Obstet Gynaecol. 2014;3(2):428-33.

11. Ozeren M, Dinc H, Ekmen U, Senakayli C, Aydemir V. Umbilical. middle cerebral artery Doppler indice in patients with preeclampsia. Eur J Obstet Gynaecol Reprod Biol. 1999;82(1):11-6.

12. Khalid M, Wahab S, Kumar V, Khalid S, Haroon S, Sabzposh NA. Doppler indices in prediction of fetal outcome in hypertensive pregnant women. NJOG. 2011;6(1):28-34.

13. Lakhkar BN, Rajgopal KN, Gourishankar PT. Doppler prediction in intra uterine growth retardation. IJRI. 2006;16(1):109-16.

14. Fleischer A, Schulman H, Farmakides G, Bracero L, Grunfeld L, Rochelson B, et al. Uterine artery doppler velocimetry in pregnant women with hypertension. Am J Obstet Gynecol. 1986;154(4):806-12.

15. Jackson MR, Walsh AJ, Morrow RJ, Muller BM, Lye SJ, Ritchie JK. Reduced placental villous tree elaboration in small-for-gestational-age pregnancies: relationship with umbilical artery Doppler waveforms. Am J Obstet Gynecol. 1995;172(2):51825.

16. Chan FY, Pun TC, Lam P, Lam C, Lee CP, Lam YH. Fetal cerebral Doppler as a predictor of perinatal outcome and subsequent neurological handicap. Obstet Gynecol. 1996;87:981-8.

17. Bhatt CJ, Arora J. Role of color Doppler in pregnancy induced hypertension (100 cases). IJRI. 2003;13(4):417-20.

18. Turan OM, Turan S, Berg C, Gembruch U, Nicolaides KH, Harman CR, et al. Duration of persistent abnormal ductus venosus flow and its impact on perinatal outcome in fetal growth restriction. Ultrasound Obstet Gynecol. 2011;38(3):295-302.

Cite this article as: Mishra R, Misra AP. Role of obstetric colour doppler studies in predicting perinatal outcome in pregnancy induced hypertension. Int J Reprod Contracept Obstet Gynecol 2020;9:1073-8. 